International Journal of Modern Physics B

(C) World Scientific Publishing Company

\title{
Classical chaotic trajectories in quantum field theory
}

\author{
Giuseppe Vitiello \\ Dipartimento di Fisica "E.R.Caianiello", Università di Salerno, 84100 Salerno, Italy \\ INFN, Gruppo Collegato di Salerno and INFM, Sezione di Salerno \\ vitiello@sa.infn.it
}

\begin{abstract}
Trajectories in the space of the unitarily inequivalent representations of the canonical commutation relations are shown to be classical trajectories. Under convenient conditions, they may exhibit properties typical of chaotic behavior in classical nonlinear dynamics. Quantum noise in fluctuating random force in the system-environment coupling and system-environment entanglement is also discussed.
\end{abstract}

Keywords: coherent states in QFT; q-deformed Hopf algebra; chaos;

\section{Introduction}

In recent years the structure of the state space in quantum field theory (QFT) has been shown 1 to be intimately related to the one of the deformed Hopf algebra 23 . This fact has been exploited to clarify some characteristic features of thermal field theories and it has been shown that the couple of "thermal" conjugate variables $\theta$ and $p_{\theta} \equiv-i \frac{\partial}{\partial \theta}$, with $\theta$ related to the $q$-deformation parameter, may describe trajectories in the space $\mathcal{H}$ of the representations, i.e. the space whose "points" are the unitarily inequivalent representations (uir) of the canonical commutation relations (ccr) 1 . In the present paper I will show that there is a symplectic structure associated to the "thermal degrees of freedom" $\theta$ and that the trajectories in the $\mathcal{H}$ space may exhibit some properties typical of chaotic trajectories in classical nonlinear dynamics.

In Section 2 I will shortly summarize the deformed Hopf algebra structure underlying QFT. In Section 3 I will comment on the quantum noise nature of the doubling of degrees of freedom implicit in the coproduct mapping of the Hopf algebra and on the entanglement between the system degrees of freedom and the doubled ones. In Section 4 I will show that trajectories in the $\mathcal{H}$ space are classical trajectories, which, under convenient conditions, may satisfy the criteria for chaoticity prescribed by nonlinear dynamics. For shortness I will not discuss possible applications of the formalism here presented.

I am pleased to dedicate to Francesco Guerra these notes on QFT: in some sense my interest in the issues here discussed is rooted in my thesis work towards the degree in Physics supervised by him at the Naples University "some" time ago. 


\section{Hopf algebra and the doubling of the degrees of freedom}

In the following I will consider the case of bosons. The conclusions, however, apply also to the fermion case.

The additivity of so-called primitive operators, such as energy, momentum, angular momentum, necessarily implies the use of the Lie-Hopf algebra in quantum theories. The prescription for operating on two modes is indeed provided by the coproduct operation, a key ingredient of Hopf algebras. The addition of, e.g., the angular momentum $J^{\alpha}, \alpha=1,2,3$, of two particles, is given by the coproduct $\Delta J^{\alpha}=J^{\alpha} \otimes \mathbf{1}+\mathbf{1} \otimes J^{\alpha} \equiv J_{1}^{\alpha}+J_{2}^{\alpha}$. The coproduct is a homomorphism which duplicates the algebra, $\Delta: \mathcal{A} \rightarrow \mathcal{A} \otimes \mathcal{A}$, i.e. $\Delta \mathcal{O}=\mathcal{O} \otimes \mathbf{1}+\mathbf{1} \otimes \mathcal{O} \equiv \mathcal{O}_{1}+\mathcal{O}_{2}$, with $\mathcal{O} \in \mathcal{A}$.

A remarkable result is that the infinitely many uir of the ccr, whose existence characterizes QFT, are classified by use of the deformed Hopf algebra. Quantum deformations of Hopf algebra have thus a deeply non-trivial physical meaning in QFT. One can indeed show 14 that the Bogolubov transformations

$$
\begin{aligned}
& A(\theta) \equiv \frac{1}{\sqrt{2}}(\alpha(\theta)+\beta(\theta))=A \cosh \theta-B^{\dagger} \sinh \theta, \\
& B(\theta) \equiv \frac{1}{\sqrt{2}}(\alpha(\theta)-\beta(\theta))=B \cosh \theta-A^{\dagger} \sinh \theta,
\end{aligned}
$$

which relate different (i.e. unitary inequivalent) representations 5 , are directly obtained by use of the deformed copodruct operation:

$$
\Delta a_{q}=a_{1} q^{1 / 2}+q^{-1 / 2} a_{2}, \quad \Delta a_{q}^{\dagger}=a_{1}^{\dagger} q^{1 / 2}+q^{-1 / 2} a_{2}^{\dagger} .
$$

Note that $\left[a_{i}, a_{j}\right]=\left[a_{i}, a_{j}^{\dagger}\right]=0, i, j=1,2, i \neq j$. In Eqs. (11) and (2) $\alpha(\theta)$ and $\beta(\theta)$ are convenient linear combinations $\frac{1}{1}$ of the coproduct operators (3) with $q=e^{2 \theta}$. Note that $\left[A(\theta), A^{\dagger}(\theta)\right]=1,\left[B(\theta), B^{\dagger}(\theta)\right]=1$ and all other commutators equal to zero. $A(\theta)$ and $B(\theta)$ also commute. The momentum suffix $\kappa$ is omitted for simplicity.

Since QFT is characterized by the existence of uir of the ccr, the intrinsic algebraic structure of QFT is thus the one of the deformed Hopf algebra.

The generator of (11) and (2) is $\mathcal{G} \equiv-i\left(A^{\dagger} B^{\dagger}-A B\right)$ :

$$
-i \frac{\delta}{\delta \theta} A(\theta)=[\mathcal{G}, A(\theta)], \quad-i \frac{\delta}{\delta \theta} B(\theta)=[\mathcal{G}, B(\theta)],
$$

and h.c.. Thus $p_{\theta} \equiv-i \frac{\delta}{\delta \theta}$ can be regarded 11 as the momentum operator "conjugate" to the "degree of freedom" $\theta$. For an assigned fixed value $\bar{\theta}$, it is

$$
\exp \left(i \bar{\theta} p_{\theta}\right) A(\theta)=\exp (i \bar{\theta} \mathcal{G}) A(\theta) \exp (-i \bar{\theta} \mathcal{G})=A(\theta+\bar{\theta}),
$$

and similarly for $B(\theta)$.

It is interesting to consider the case of time-dependent deformation parameter. In such a case, the Heisenberg equation for $A(t, \theta(t))$ is

$$
-i \dot{A}(t, \theta(t))=-i \frac{\delta}{\delta t} A(t, \theta(t))-i \frac{\delta \theta}{\delta t} \frac{\delta}{\delta \theta} A(t, \theta(t))=
$$




$$
[H, A(t, \theta(t))]+\frac{\delta \theta}{\delta t}[\mathcal{G}, A(t, \theta(t))]=[H+Q, A(t, \theta(t))],
$$

and $Q \equiv \frac{\delta \theta}{\delta t} \mathcal{G}$ plays the role of the heat-term in dissipative systems. $H$ is the Hamiltonian responsible for the time variation in the explicit time dependence of $A(t, \theta(t)) . H+Q$ can be therefore identified with the free energy $[6$ ? variations in time of the deformation parameter involve dissipation.

Let $|0\rangle \equiv|0\rangle \otimes|0\rangle$ denote the vacuum annihilated by $A$ and $B, A|0\rangle=0=B|0\rangle$. By introducing the suffix $\kappa$, at finite volume $V$ one obtains

$$
|0(\theta)\rangle=\exp \left(i \sum_{\kappa} \theta_{\kappa} \mathcal{G}_{\kappa}\right)|0\rangle=\prod_{k} \frac{1}{\cosh \theta_{k}} \exp \left(\tanh \theta_{k} A_{k}^{\dagger} B_{k}^{\dagger}\right)|0\rangle .
$$

Here $\theta$ denotes the set $\left\{\theta_{\kappa}=\frac{1}{2} \ln q_{\kappa}, \forall \kappa\right\}$ and $\langle 0(\theta) \mid 0(\theta)\rangle=1$.

The vacuum $|0(\theta)\rangle$ is an $S U(1,1)$ generalized coherent state ${ }^{7}$ (the group structure actually is $\left.\bigotimes_{\kappa} S U(1,1)_{\kappa}\right)$ : coherence and the vacuum structure in QFT are thus intrinsically related to the deformed Hopf algebra. In the following $\mathcal{H}_{\theta}$ will denote the Hilbert space with vacuum $|0(\theta)\rangle: \mathcal{H}_{\theta} \equiv\{|0(\theta)\rangle\}$.

In the infinite volume limit, the number of degrees of freedom becomes uncountable infinite, hence one obtains $\frac{68}{8}\left\langle 0(\theta) \mid 0\left(\theta^{\prime}\right)\right\rangle \rightarrow 0 \quad$ as $V \rightarrow \infty, \quad \forall \theta, \theta^{\prime}, \theta \neq \theta^{\prime}$, which means that the Hilbert spaces $\mathcal{H}_{\theta}$ and $\mathcal{H}_{\theta^{\prime}}$ become unitarily inequivalent. In this limit, the "points" of the space $\mathcal{H} \equiv\left\{\mathcal{H}_{\theta}, \forall \theta\right\}$ of the infinitely many uir of the ccr are labelled by the deformation parameter $\theta 146$.

\section{Quantum noise and entanglement}

The doubling of the degrees of freedom which, as we have seen, is built in in the algebraic structure of QFT, is rich of physical meanings (e.g. in thermal field theory the doubled degree of freedom describes the heat bath 16 ; ; or, near a black hole, such a doubling describes the modes on the two sides of the horizon 9 ).

The doubling of the degrees of freedom can also be formally understood by considering the standard expression for the Wigner function 10,

$$
W(p, x, t)=\frac{1}{2 \pi \hbar} \int \psi^{*}\left(x-\frac{1}{2} y, t\right) \psi\left(x+\frac{1}{2} y, t\right) e^{\left(-i \frac{p y}{\hbar}\right)} d y .
$$

By using $x_{ \pm}=x \pm \frac{1}{2} y$, the associated density matrix function is

$$
W(x, y, t)=\left\langle x_{+}|\rho(t)| x_{-}\right\rangle=\psi^{*}\left(x_{-}, t\right) \psi\left(x_{+}, t\right) .
$$

The density matrix and the Wigner function formalism thus requires the introduction of a "doubled" set of coordinates, $\left(x_{ \pm}, p_{ \pm}\right)$(or $\left(x, p_{x}\right)$ and $\left.\left(y, p_{y}\right)\right)$. Such a doubling can be shown 11 to coincide with the one discussed in Sec. 2.

The doubled degrees of freedom play the role of the thermal bath (the environment) degrees of freedom $[8$. In the frame of the formalism by Schwinger 12 and by Feynman and Vernon 13 for the quantum Brownian motion it is possible to show 11 that the role of the "doubled" $y$ coordinate is absolutely crucial in the quantum 
regime, since there it accounts for the quantum noise in the fluctuating random force in the system-environment coupling: in the limit of $y \rightarrow 0$ (i.e. for $x_{+}=x_{-}$) quantum effects are lost and the classical limit is obtained.

I now remark that the state $|0(\theta)\rangle$ in Eq. (7) is an entangled state. The entanglement between the modes $A$ and $B$ is indeed manifest by writing

$$
|0(\theta)\rangle=\left(\prod_{k} \frac{1}{\cosh \theta_{k}}\right)\left(|0\rangle \otimes|0\rangle+\sum_{k} \tanh \theta_{k}\left(\left|A_{k}\right\rangle \otimes\left|B_{k}\right\rangle\right)+\ldots\right),
$$

which clearly cannot be factorized into the product of two single-mode states. By defining $|\mathcal{I}\rangle \equiv \exp \left(\sum_{\kappa} A_{\kappa}^{\dagger} B_{\kappa}^{\dagger}\right)|0\rangle$, the state $|0(\theta)\rangle$ may be also written as:

$$
\begin{gathered}
|0(\theta)\rangle=\exp \left(-\frac{1}{2} S_{A}\right)|\mathcal{I}\rangle=\exp \left(-\frac{1}{2} S_{B}\right)|\mathcal{I}\rangle, \\
S_{A} \equiv-\sum_{\kappa}\left\{A_{\kappa}^{\dagger} A_{\kappa} \ln \sinh ^{2} \theta_{\kappa}-A_{\kappa} A_{\kappa}^{\dagger} \ln \cosh ^{2} \theta_{\kappa}\right\} .
\end{gathered}
$$

$S_{B}$ is given by a similar expression with $B_{\kappa}$ and $B_{\kappa}^{\dagger}$ replacing $A_{\kappa}$ and $A_{\kappa}^{\dagger}$, respectively. I simply write $S$ for either $S_{A}$ or $S_{B}$. I can also write 68 .

$$
\begin{aligned}
|0(\theta)\rangle & =\sum_{n=0}^{+\infty} \sqrt{W_{n}}(|n\rangle \otimes|n\rangle), \\
W_{n} & =\prod_{k} \frac{\sinh ^{2 n_{k}} \theta_{k}}{\cosh ^{2\left(n_{k}+1\right)} \theta_{k}},
\end{aligned}
$$

with $n$ denoting the set $\left\{n_{\kappa}\right\}$ and with $0<W_{n}<1$ and $\sum_{n=0}^{+\infty} W_{n}=1$. Then

$$
\left\langle 0(\theta)\left|S_{A}\right| 0(\theta)\right\rangle=\sum_{n=0}^{+\infty} W_{n} \ln W_{n},
$$

and thus $S$ can be interpreted as the entropy operator $\underline{68}$ and it provides a measure of the degree of entanglement. I remark that the entanglement is truly realized in the infinite volume limit where

$$
\langle 0(\theta) \mid 0\rangle=e^{-\frac{V}{(2 \pi)^{3}} \int d^{3} \kappa \ln \cosh \theta_{\kappa}} \underset{V \rightarrow \infty}{\longrightarrow} 0,
$$

provided $\int d^{3} \kappa \ln \cosh \theta_{\kappa}$ is not identically zero. The probability of having the component state $|n\rangle \otimes|n\rangle$ in the state $|0(\theta)\rangle$ is $W_{n}$. Since $W_{n}$ is a decreasing monotonic function of $n$, the contribution of the states $|n\rangle \otimes|n\rangle$ would be suppressed for large $n$ at finite volume. In that case, the transformation induced by the unitary operator $G^{-1}(\theta) \equiv \exp \left(-i \sum_{\kappa} \theta_{\kappa} \mathcal{G}_{\kappa}\right)$ could disentangle the $A$ and $B$ sectors. However, this is not the case in the infinite volume limit, where the summation extends to an infinite number of components and Eq. (16) holds (in such a limit Eq. (7) is only a formal relation since $G^{-1}(\theta)$ does not exist as a unitary operator).

It is interesting to note that, although the mode $B$ is related with quantum noise effects, nevertheless the $A-B$ (or system-environment) entanglement is not 
affected by such noise effects. The robustness of the entanglement is rooted in the fact that, once the infinite volume limit is reached, there is no unitary generator able to disentangle the system-environment coupling.

\section{Chaotic behavior of the trajectories in the $\mathcal{H}$ space}

In order to discuss the chaotic behavior under certain conditions of the trajectories in the $\mathcal{H}$ space, it is useful to recall some of the features of the $S U(1,1)$ group structure. See, e.g., 7 for more details.

$S U(1,1)$ realized on $C \times C$ consists of all unimodular $2 \times 2$ matrices leaving invariant the Hermitian form $\left|z_{1}\right|^{2}-\left|z_{2}\right|^{2}, z_{i} \in C, i=1,2$. The complex $z$ plane is foliated under the group action into three orbits: $X_{+}=\{z:|z|<1\}, X_{-}=\{z$ : $|z|>1\}$ and $X_{0}=\{z:|z|=1\}$.

It may be shown that the unit circle $X_{+}=\{\zeta:|\zeta|<1\}, \zeta \equiv e^{i \phi} \tanh \theta$, is isomorphic to the upper sheet of the hyperboloid which is the set $\mathbf{H}$ of pseudoEuclidean bounded (unit norm) vectors $\mathbf{n}: \mathbf{n} \cdot \mathbf{n}=1$. $\mathbf{H}$ is a Kählerian manifold with metrics $d s^{2}=4 \frac{\partial^{2} F}{\partial \zeta \partial \zeta} d \zeta \cdot d \bar{\zeta}$. Here $F \equiv-\ln \left(1-|\zeta|^{2}\right)$ is the Kählerian potential and the metric is invariant under the group action.

The Kählerian manifold $\mathbf{H}$ is known to have a symplectic structure and thus it may be considered as the phase space for the classical dynamics generated by the group action 7 .

The $S U(1,1)$ generalized coherent states are recognized to be "points" in $\mathbf{H}$ and transitions among these points induced by the group action are therefore classical trajectories 7 in $\mathbf{H}$ (a similar situation occurs 7 in the $S U(2)$ (fermion) case).

The above considerations thus show that the space of the unitarily inequivalent representations of the ccr is a Kählerian manifold, $\mathcal{H} \equiv\left\{\mathcal{H}_{\theta}, \forall \theta\right\} \approx \mathbf{H}$, namely it has a symplectic structure and a classical dynamics is established on it by the $S U(1,1)$ action (generated by $\mathcal{G}$ or by $p_{\theta}$ in the notation of Section $1: \mathcal{H}_{\theta} \rightarrow \mathcal{H}_{\theta^{\prime}}$ ): Trajectories in $\mathcal{H}$ describe transitions through the representations $\mathcal{H}_{\theta}=\{|0(\theta)\rangle\}$ as the $\theta$-parameter changes (i.e. through the physical phases of the system, the system order parameter being dependent on $\theta$ ). One may then assume time-dependent $\theta$ : $\theta=\theta(t)$. For example, this is the case of dissipative systems and of non-equilibrium thermal field theories where $\theta_{\kappa}=\theta_{\kappa}[\beta(t)]$, with $\beta$ the inverse time-dependent temperature.

In conclusion, the group action induces classical trajectories in $\mathcal{H}$. Such a result has been also obtained elsewhere 14115 on the ground of more phenomenological considerations.

In the following it will be convenient to use the notation $|0(t)\rangle_{\theta} \equiv|0(\theta(t))\rangle$. For any $\theta(t)=\left\{\theta_{\kappa}(t), \forall \kappa\right\}$ it is

$$
{ }_{\theta}\langle 0(t) \mid 0(t)\rangle_{\theta}=1, \quad \forall t
$$

I will now restrict the discussion to the case in which $\theta_{\kappa}(t)$ is, for any $\kappa$, a growing 
function of time and

$$
\theta(t) \neq \theta\left(t^{\prime}\right), \quad \forall t \neq t^{\prime}, \quad \text { and } \quad \theta(t) \neq \theta^{\prime}\left(t^{\prime}\right), \quad \forall t, t^{\prime} .
$$

Under such conditions, as shown below, the trajectories in $\mathcal{H}$ satisfy the requirements for chaotic behavior in classical nonlinear dynamics. These requirements can be formulated as follows 16 .

i) the trajectories are bounded and each trajectory does not intersect itself.

ii) trajectories specified by different initial conditions do not intersect.

iii) trajectories of different initial conditions are diverging trajectories.

Let the initial time be $t_{0}=0$. The trajectory "initial condition" is then specified by the $\theta(0)$-set, $\theta(0)=\left\{\theta_{\kappa}(0), \forall \kappa\right\}$. One obtains

$$
{ }_{\theta}\left\langle 0(t) \mid 0\left(t^{\prime}\right)\right\rangle_{\theta} \underset{V \rightarrow \infty}{\longrightarrow} 0, \quad \forall t, t^{\prime}, \quad \text { with } \quad t \neq t^{\prime},
$$

provided $\int d^{3} \kappa \ln \cosh \left(\theta_{\kappa}(t)-\theta_{\kappa}\left(t^{\prime}\right)\right)$ is finite and positive for any $t \neq t^{\prime}$. Eq. (19) expresses the unitary inequivalence of the states $|0(t)\rangle_{\theta}$ (and of the associated Hilbert spaces $\left\{|0(t)\rangle_{\theta}\right\}$ ) at different time values $t \neq t^{\prime}$ in the infinite volume limit. The non-unitarity of time evolution implied for example by the damping is consistently recovered in the unitary inequivalence among representations $\left\{|0(t)\rangle_{\theta}\right\}$ 's at different $t$ 's in the infinite volume limit.

The trajectories are bounded in the sense of Eq. (17), which shows that the "length" (the norm) of the "position vectors" (the state vectors at time $t$ ) in $\mathcal{H}$ is finite (and equal to one) for each $t$. Eq. (17) rests on the invariance of the Hermitian form $\left|z_{1}\right|^{2}-\left|z_{2}\right|^{2}, z_{i} \in C, i=1,2$ and I also recall that the manifold of points representing the coherent states $|0(t)\rangle_{\theta}$ for any $t$ is isomorphic to the product of circles of radius $r_{\kappa}^{2}=\tanh ^{2}\left(\theta_{\kappa}(t)\right)$ for any $\kappa$, as mentioned above.

I also observe that Eq. (19) expresses the fact that the trajectory does not crosses itself as time evolves (it is not a periodic trajectory): the "points" $\left\{|0(t)\rangle_{\theta}\right\}$ and $\left\{\left|0\left(t^{\prime}\right)\right\rangle_{\theta}\right\}$ through which the trajectory goes, for any $t$ and $t^{\prime}$, with $t \neq t^{\prime}$, after the initial time $t_{0}=0$, never coincide. The requirement $i$ ) is thus satisfied.

In the infinite volume limit, we also have

$$
{ }_{\theta}\left\langle 0(t) \mid 0\left(t^{\prime}\right)\right\rangle_{\theta^{\prime}} \underset{V \rightarrow \infty}{\longrightarrow} 0 \quad \forall t, t^{\prime} \quad, \quad \forall \theta \neq \theta^{\prime} .
$$

Notice that, under the assumption (18), Eq. (20) is true also for $t=t^{\prime}$. The meaning of Eqs. (20) is that trajectories specified by different initial conditions $\theta(0) \neq \theta^{\prime}(0)$ never cross each other. The requirement ii) is thus satisfied.

Let me now study how the "distance" between trajectories in the space $\mathcal{H}$ behaves as time evolves. I consider two trajectories of slightly different initial conditions, say $\theta^{\prime}(0)=\theta(0)+\delta \theta$, with small $\delta \theta$. A difference between the states $|0(t)\rangle_{\theta}$ and $|0(t)\rangle_{\theta^{\prime}}$ is the one between the respective expectation values of the number operator $A_{\kappa}^{\dagger} A_{\kappa}$. We have, for any $\kappa$ at any given $t$,

$$
\begin{aligned}
\Delta \mathcal{N}_{A_{\kappa}}(t) \equiv & \mathcal{N}^{\prime}{ }_{A_{\kappa}}\left(\theta^{\prime}(t)\right)-\mathcal{N}_{A_{\kappa}}(\theta(t))={ }_{\theta^{\prime}}\left\langle 0(t)\left|A_{\kappa}^{\dagger} A_{\kappa}\right| 0(t)\right\rangle_{\theta^{\prime}-{ }_{\theta}\left\langle 0(t)\left|A_{\kappa}^{\dagger} A_{\kappa}\right| 0(t)\right\rangle_{\theta}} \\
& =\sinh ^{2}\left(\theta^{\prime}{ }_{\kappa}(t)\right)-\sinh ^{2}\left(\theta_{\kappa}(t)\right)=\sinh \left(2 \theta_{\kappa}(t)\right) \delta \theta_{\kappa}(t),
\end{aligned}
$$


where $\delta \theta_{\kappa}(t) \equiv \theta^{\prime}{ }_{\kappa}(t)-\theta_{\kappa}(t)$ is assumed to be greater than zero, and the last equality holds for "small" $\delta \theta_{\kappa}(t)$ for any $\kappa$ at any given $t$. Then, by assuming that $\frac{\partial \delta \theta_{\kappa}}{\partial t}$ has negligible variations in time, the time-derivative gives

$$
\frac{\partial}{\partial t} \Delta \mathcal{N}_{A_{\kappa}}(t)=2 \frac{\partial \theta_{\kappa}(t)}{\partial t} \cosh \left(2 \theta_{\kappa}(t)\right) \delta \theta_{\kappa},
$$

which shows that, provided $\theta_{\kappa}(t)$ is a growing function of $t$, small variations in the initial conditions lead to growing in time $\Delta \mathcal{N}_{A_{\kappa}}(t)$, namely to diverging trajectories as time evolves.

In the assumed hypothesis, at enough large $t$ the divergence is dominated by $\exp \left(2 \theta_{\kappa}(t)\right)$. For each $\kappa$, the quantity $2 \theta_{\kappa}(t)$ could be thus thought to play the role similar to the one of the Lyapunov exponent.

I also observe that, since ${ }^{6} \sum_{\kappa} E_{\kappa} \dot{\mathcal{N}}_{A_{\kappa}} d t=\frac{1}{\beta} d S_{A}$, where $E_{\kappa}$ is the energy of the mode $A_{\kappa}, d S_{A}$ is the entropy variation associated to the modes $A$ and $\dot{\mathcal{N}}_{A_{\kappa}}$ denotes the time derivative of $\mathcal{N}_{A_{\kappa}}$, the divergence of trajectories of different initial conditions may be expressed in terms of differences in the variations of the entropy (cf. Eqs. (21) and (22)):

$$
\Delta \sum_{\kappa} E_{\kappa} \dot{\mathcal{N}}_{A_{\kappa}}(t) d t=\frac{1}{\beta}\left(d S_{A}^{\prime}-d S_{A}\right)
$$

In conclusion, also the requirement iii) is satisfied. The trajectories in the $\mathcal{H}$ space thus exhibit, under the condition (18), properties typical of the chaotic behavior in classical nonlinear dynamics.

\section{Conclusions}

The deformed Hopf algebra structure of QFT implies the doubling of the system degrees of freedom. The doubled degrees of freedom play the role of the thermal bath or environment degrees of freedom and are entangled with the system degrees of freedom. They also account for quantum noise in the fluctuating random forces in the system-environment coupling. In such a frame, the trajectories in the space of the representations of the canonical commutation relations turn out to be classical trajectories and, under convenient conditions, they may exhibit properties typical of classical chaotic trajectories in nonlinear dynamics. Finally, it might be worth to remark that quantum noise effects accounted for by the doubled degrees of freedom are not the source of such a chaotic behavior.

\section{Acknowledgements}

Partial financial support from Murst, INFN, INFM and the ESF Program COSLAB is acknowledged.

\section{References}

1. E. Celeghini, S. De Martino, S. De Siena, A. Iorio, M. Rasetti and G. Vitiello, Phys. Lett. A 244, 455 (1998); Annals Phys. 241, 50 (1995). 
2. V. G. Drinfeld, in Proc. ICM Berkeley, CA, A. M. Gleason, ed.; AMS, Providence, R.I., 1986, 798p.

M. Jimbo, Int. J. Mod. Phys. A 4, 3759 (1989).

Yu. I. Manin, Quantum groups and Non-Commutative Geometry, CRM, Montreal, 1988.

3. E. Celeghini, T. D. Palev and M. Tarlini, Mod. Phys. Lett. B 5, 187 (1991).

P. P. Kulish and N. Y. Reshetikhin, Lett. Math. Phys. 18, 143 (1989).

4. A. Iorio and G. Vitiello, Mod. Phys. Lett. B 8, 269 (1994); Annals Phys. 241, 496 (1995)

5. O. Bratteli and D. W. Robinson, Operator Algebras and Quantum Statistical Mechanics, Springer, Berlin, 1979.

6. E. Celeghini, M. Rasetti and G. Vitiello, Annals Phys. 215, 156 (1992).

7. A. Perelomov, Generalized Coherent States and Their Applications, Springer, Berlin, 1986.

8. Y. Takahashi and H. Umezawa, Collective Phenomena 2, 55 (1975).

H. Umezawa, Advanced field theory: micro, macro and thermal concepts, AIP, N.Y. 1993.

9. M. Martellini, P. Sodano and G. Vitiello, Nuovo Cim. A 48, 341 (1978).

A. Iorio, G. Lambiase and G. Vitiello, arXiv:hep-th/0204034

10. R. P. Feynman, Statistical Mechanics, W. A. Benjamin Publ. Co., Reading, Ma. 1972

11. Y. N. Srivastava, G. Vitiello and A. Widom, Annals Phys. 238, 200 (1995)

12. J. Schwinger, J. Math. Phys. 2, 407 (1961)

13. R. P. Feynman and F. L. Vernon, Annals Phys. 24, 118 (1963)

14. R. Manka, J. Kuczynski and G. Vitiello, Nucl. Phys. B 276, 533 (1986).

15. E. Del Giudice, R. Manka, M. Milani and G. Vitiello, Phys. Lett. A 206, 661 (1988).

16. R. Hilborn, Chaos and nonlinear Dynamics, Oxford University Press, Oxford 1994 